\title{
Clinical impression and Western Aphasia Battery classification of aphasia in acute ischemic stroke: Is there a discrepancy?
}

\author{
Aju Abraham John, Mahendra Javali, Rohan Mahale, Anish Mehta, P. T. Acharya, R. Srinivasa \\ Department of Neurology, M.S. Ramaiah Medical College and Hospital, Bengaluru, Karnataka, India
}

\begin{abstract}
Background: Language disturbance is a common symptom of stroke, a prompt identifier of the event, and can cause devastating cognitive impairments. There are many inconsistencies and discrepancies between the different methods used for its evaluation. The relationship between Western Aphasia Battery (WAB) and a simple bedside clinical examination is not clear. Aim: The aim of this study is to determine if bedside clinical impression of aphasia type can reliably predict WAB classification of aphasia and to describe the discrepancies between them. Materials and Methods: Eighty-two consecutive cases of acute ischemic stroke and aphasia were evaluated with bedside aphasia assessment, handedness by Edinburgh Handedness Inventory and WAB scoring was done. Kappa statistics was used to find the overall agreement of clinical impression and WAB. Results: Disagreement was seen predominantly for the nonfluent aphasias when the clinical impression was compared with WAB classification. WAB also had diagnosed three cases as having anomic aphasia using taxonomic classification, but same cases had normal language by aphasia quotient scoring of WAB. There was an overall agreement of $63.4 \%$ between patient's bedside clinical impression and WAB classification of aphasia, with a $P<0.001$. Conclusion: Clinical impression was fairly reliable, as compared to WAB in assessing the type of aphasia. Clinical impression was appropriate in an acute setting, but WAB was required to quantify the severity of deficit, which may help in accessing prognosis, monitoring progression, and rehabilitation planning. Along with WAB, a bedside clinical impression should be done for all the patients to strengthen the description of aphasic deficit.
\end{abstract}

Key words: Aphasia, clinical impression, ischemic stroke

\section{Introduction}

Aphasia is one of the most common and devastating cognitive impairments of stroke. ${ }^{[1,2]}$ About $21-38 \%$ of acute stroke patients experience aphasia. ${ }^{[3]}$ The Western Aphasia Battery (WAB) is one of the commonly used batteries to evaluate language function and has high internal consistency, test-retest reliability, and validity. ${ }^{[4]}$ WAB can determine the presence, type, and severity

\section{Address for correspondence:}

Prof. R. Srinivasa, Department of Neurology,

M.S. Ramiah Medical College and Hospital, New BEL Road,

MSR Nagar, Bengaluru - 560 054, Karnataka, India.

E-mail: ajujohn999@gmail.com

\begin{tabular}{|l|l|}
\hline \multicolumn{2}{|c|}{ Access this article online } \\
\hline Quick Response Code: & Website: \\
\hline & www.ruralneuropractice.com \\
\cline { 2 - 2 } & \\
\hline & \\
\hline
\end{tabular}

of aphasia. It evaluates language profiles for fluency, comprehension, repetition, and naming. ${ }^{[5]}$

Most speech pathologists use different batteries for language assessment. However, they are lengthy, time-consuming, and require neurologists or trained speech and language pathologists. They may not be practical in an acute setting where a rapid evaluation and treatment is required. To best of our knowledge, there are only few reports of the accuracy of WAB to bedside clinical impression, especially in Indian patients. Most of the studies earlier are done in sub-acute or recovery phase of stroke. Hence, this study was undertaken to compare the reliability of WAB to bedside clinical impression of aphasia in acute phase ischemic stroke.

This is an open access article distributed under the terms of the Creative Commons Attribution-NonCommercial-ShareAlike 3.0 License, which allows others to remix, tweak, and build upon the work non-commercially, as long as the author is credited and the new creations are licensed under the identical terms.

For reprints contact: reprints@medknow.com

How to cite this article: John AA, Javali M, Mahale $R$, Mehta A, Acharya PT, Srinivasa R. Clinical impression and western aphasia battery classification of aphasia in acute ischemic stroke: Is there a discrepancy?. J Neurosci Rural Pract 2017;8:74-8. 


\section{Materials and Methods}

A total of 295 consecutive patients were screened for aphasia following an acute ischemic stroke from November 2013 to February 2016. Patients without aphasia, altered level of sensorium, psychiatric illness, dementia, or recent head injury were excluded from this study. Eighteen cases with probable aphasia were also excluded due to reduced sustained attention following the stroke, and a total of 82 patients were recruited for the study. All the patients were examined within $48 \mathrm{~h}$ of admission to the hospital and 64 of them within $24 \mathrm{~h}$.

Clinical impression of aphasia was done at bedside using a standard protocol. Spontaneous speech was accessed for articulation, fluency, and presence of paraphasias. Comprehension was tested with yes or no questions, pointing commands, and one to three step commands. Naming was evaluated for objects, object parts, body parts, and colors. Repetition was done for single words to complex sentences. The level of adequacy for reading and writing were also tested. WAB was administered simultaneously in all the patients. Subscores on fluency, comprehension, repetition, and naming impairment were classified according to the taxonomic table [Table 1]. Accordingly, aphasia can be classified into global aphasia (GA), Broca's aphasia (BA), Wernicke's aphasia (WA), transcortical motor (TCM), transcortical sensory (TCS), mixed transcortical aphasia (MTCA), conduction aphasia (CA), and anomic aphasia (AA) types. In addition, the WAB describes severities of aphasia as aphasia quotients (AQ). ${ }^{[5]}$ The severity of aphasia was calculated with AQ of $\mathrm{WAB}$ and language was classified as normal if an AQ of 93.8 or above was achieved. The evaluation was done by two neurologists and two trained speech and language pathologists. Kannada (regional language) version of WAB was administered to patients not fluent in English, which is standardized by a study done by Chengappa and Kumar. ${ }^{[6]}$

Table 1: Taxonomic table of Western Aphasia Battery (Kertesz, 1981)

\begin{tabular}{lcccc}
\hline & \multicolumn{4}{c}{ Criteria for classification } \\
\cline { 2 - 5 } & Fluency & Comprehension & Repetition & Naming \\
\hline Global & $0-4$ & $0-3.9$ & $0-4.9$ & 0 to6 \\
Broca's & $0-4$ & $4-10$ & $0-7.9$ & $0-8$ \\
Wernicke's & $0-4$ & $0-3.9$ & $5-10$ & $0-6$ \\
$\begin{array}{l}\text { Transcortical } \\
\text { motor }\end{array}$ & $0-4$ & $4-10$ & $8-10$ & $0-8$ \\
$\begin{array}{l}\text { Transcortical } \\
\text { sensory }\end{array}$ & $5-10$ & $0-6.9$ & $0-7.9$ & $0-9$ \\
$\begin{array}{l}\text { Mixed } \\
\text { transcortical }\end{array}$ & $5-10$ & $0-6.9$ & $8-10$ & $0-9$ \\
$\begin{array}{l}\text { Conduction } \\
\text { Anomic }\end{array}$ & $5-10$ & $7-10$ & $0-6.9$ & $0-9$ \\
\hline
\end{tabular}

This is a prospective observational study and no new treatments, or novel therapeutic interventions were used. Institutional ethical committee permission was taken for this study. SPSS Inc. Released 2009, PASW Statistics for Windows, Version 18.0. Chicago: SPSS Inc., was used to analyze the data. Descriptive statistics was used for analysis of different type of aphasia according to clinical impression and WAB and presented as percentages. Kappa statistics was used to find the overall agreement between clinical impression and WAB classification. $P<0.05$ is taken for statistical significance.

\section{Results}

All the patients were right-handed in this study. All of them had a middle cerebral artery territory infarction. There was an overall agreement of $63.4 \%$ between clinical impression and WAB classification of aphasia (kappa score of $0.504, P<0.001)$. The cross tabulation of WAB type of aphasia and clinical impression is shown in Table 2.

Forty-two cases were classified as having GA by clinical impression. Of these, only 28 was GA by WAB classification. Out of remaining 14 cases of GA by clinical impression, eight were $\mathrm{BA}$; five were $\mathrm{WA}$, and one was MTCA by WAB classification. Of the 19 cases of BA by clinical impression, only seven were BA by WAB. Of the remaining 12 cases, ten were $\mathrm{AA}$ and two turned out to be TCM by WAB classification. Clinical impression failed to recognize most cases of $\mathrm{AA}$, but WAB detected 14 cases, of which ten were BA and two were WA as per the clinical impression. WAB diagnosed three cases of $\mathrm{AA}$ as having aphasia by the taxonomic classification, but same cases had normal language by AQ (AQ more than 93.8). Both clinical impression and WAB diagnosed one case each of TCM and TCS Aphasia accurately, but its significance cannot be commented due to small sample size. Clinical impression missed two cases each of TCM and TCS Aphasia.

\section{Discussion}

Clinical impression agreed with WAB classification of aphasia (AQ of more than 93.8) in most patients for normal language. Only three patients $(3.6 \%)$ with AA by WAB had normal language by bedside examination. Clinical impression correlated with WAB classification in 52 cases (63.4\%). A similar study done by, Carol et al., showed a correlation of $54 \% .{ }^{[7]}$ Discrepancies in aphasia classification were seen in the remaining thirty cases (36.6\%).

Agreement of clinical impression with WAB according to the type of aphasia is shown in Figure 1. Similar 
Table 2: Cross tabulation of Western Aphasia Battery and clinical impression

\begin{tabular}{|c|c|c|c|c|c|c|c|c|c|}
\hline \multirow[t]{2}{*}{ Type** } & \multicolumn{8}{|c|}{ Clinical impression (\%) } & \multirow[t]{2}{*}{ Total } \\
\hline & GA & BA & WA & TCM & TCS & MTCA & CA & AA & \\
\hline \multicolumn{10}{|l|}{$\mathrm{WAB}^{*}$} \\
\hline GA & $28(100)$ & $0(0)$ & $0(0)$ & $0(0)$ & $0(0)$ & $0(0)$ & $0(0)$ & $0(0)$ & $28(100)$ \\
\hline BA & $8(53.3)$ & $7(46.7)$ & $0(0)$ & $0(0)$ & $0(0)$ & $0(0)$ & $0(0)$ & $0(0)$ & $15(100)$ \\
\hline WA & $5(29.4)$ & 0.0 & $12(70.6)$ & $0(0)$ & $0(0)$ & $0(0)$ & $0(0)$ & $0(0)$ & $17(100)$ \\
\hline TCM & $0(0)$ & $2(66.7)$ & $0(0)$ & $1(33.3)$ & $0(0)$ & $0(0)$ & $0(0)$ & $0(0)$ & $3(100)$ \\
\hline TCS & $0(0)$ & $0(0)$ & $2(66.7)$ & $0(0)$ & $1(33.3)$ & $0(0)$ & $0(0)$ & $0(0)$ & $3(100)$ \\
\hline MTCA & $1(100)$ & $0(0)$ & $0(0)$ & $0(0)$ & $0(0)$ & $0(0)$ & $0(0)$ & $0(0)$ & $1(100)$ \\
\hline $\mathrm{CA}$ & $0(0)$ & $0(0)$ & $0(0)$ & $0(0)$ & $0(0)$ & $0(0)$ & $1(100)$ & $0(0)$ & $1(100)$ \\
\hline AA & 0.0 & $10(71.4)$ & $2(14.3)$ & $0(0)$ & $0(0)$ & $0(0)$ & $0(0)$ & $2(14.3)$ & $14(100)$ \\
\hline Total & 42 (51.2) & $19(23.2)$ & $16(19.5)$ & $1(1.2)$ & $1(1.2)$ & $0(0)$ & $1(1.2)$ & $2(2.4)$ & $82(100)$ \\
\hline
\end{tabular}

${ }^{*}$ WAB: Western Aphasia Battery, ${ }^{* *}$ GA: Global aphasia, BA: Brocas aphasia, WA: Wernickes aphasia, TCM: Transcortical motor aphasia, TCS: Transcortical sensory aphasia, MTCA: Mixed transcortical aphasia, CA: Conduction aphasia, AA: Anomic aphasia

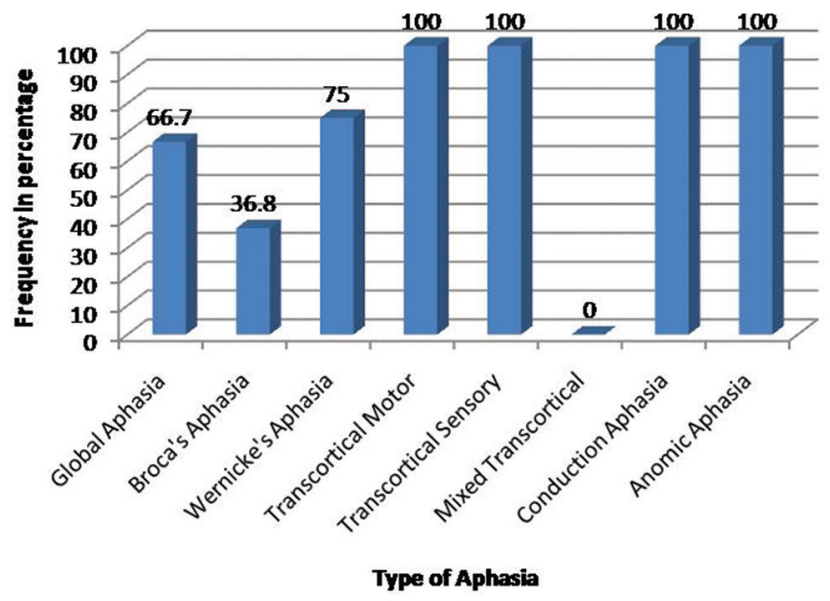

Figure 1: Clinical impression agreement with Western Aphasia Battery in percentages

agreement of WAB with clinical impression is shown in Figure $2.66 .7 \%$ cases of GA by clinical impression were in agreement with WAB. Among the misdiagnosed cases of GA, $19 \%$ of these cases turned out to be BA and $11.9 \%$ was WA by WAB classification. $75 \%$ of WA, diagnosed by clinical impression was in agreement with WAB but only $36.8 \%$ of BA cases correlated with $\mathrm{WAB}$, as shown in the cross-tabulation table [Table 2]. The misdiagnosed cases of BA turned out to be mainly AA (52.6\%) and TCM $(10.5 \%)$ in the remaining. Disagreement was seen predominantly for AA patients whom WAB was able to diagnose, but clinical impression could detect only $14.3 \%$ (2 patients of 14) accurately. Most of these cases had a near normal AQ with scores below nine only in naming, with other subtests within normal limits. Clinical impression showed $71.4 \%$ of these AA cases by WAB to be BA. This disparity of AA classification was significant in this study. Clinical diagnosis disagreed over the majority $(85.7 \%)$ of AA cases by WAB type. This discrepancy may have been due to paraphasic errors on naming subtest of WAB or probable apraxia of speech, which was not detected by bedside examination. A study

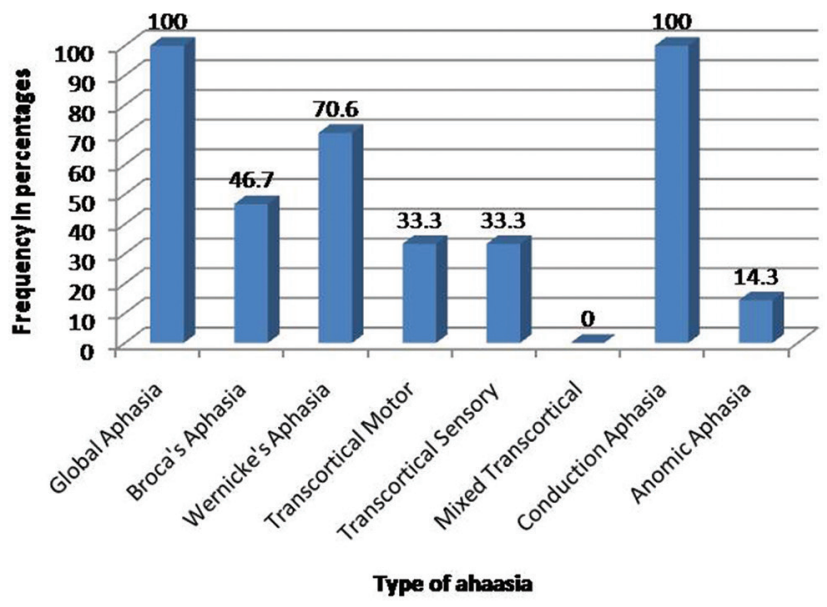

Figure 2: Western Aphasia Battery agreement with clinical impression in percentages

by Carol et al. also showed similar findings where clinical impression disagreed over more than half of the subjects for WAB's anomic classification. They retrospectively found most subjects to have a coexisting apraxia of speech, and those subjects were clinically described as normal..$^{[7]}$

Of the transcortical aphasias, each case of TCM and TCS by clinical impression was in agreement with WAB. But WAB detected two more cases of TCM and TCS each, which was classified as BA and WA by clinical impression respectively. One case of MTCA by WAB was diagnosed as GA by clinical impression. The single case of CA by clinical impression was in agreement with WAB. The significance of correlation between TCM, TCS, MTCA, and CA types could not be commented as their sample size was limited.

On comparing the fluent and nonfluent aphasias, there was more agreement for fluent aphasias by clinical impression to WAB classification as shown in Figures 3 and 4 . There was a $75 \%$ versus $36.8 \%$ and 


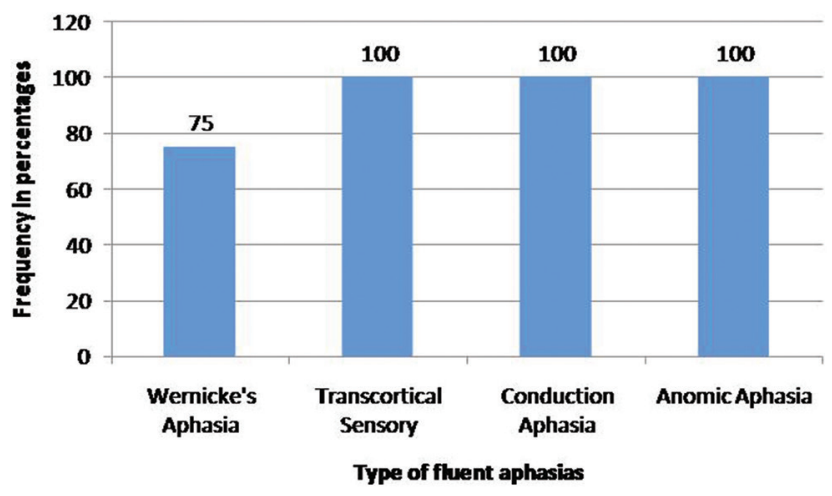

Figure 3: Agreement of clinical impression with Western Aphasia Battery in fluent aphasias

$66.7 \%$ agreement for WA as compared to BA and GA, respectively. All cases of TCS, CA, and AA diagnosed by clinical impression correlated with WAB, but WAB detected more AA than clinical impression. This was in contrast to the study by Carol et al. where more agreement was seen for the fluent classifications. ${ }^{[7]}$ Another discrepancy was also noted in the classification of AA. Three patients were diagnosed as AA by WAB with a naming score of $<9$, with other subtest scores being normal. The interesting aspect is that they had an AQ of more than 93.8, which describes them as having normal language. They had both AA and normal language by WAB, so it becomes conflicting to label them as aphasic or nonaphasic. These three patients had normal language by clinical impression.

It has been said that classification of aphasia is likely to be influenced by biases, adequate training, type of battery used, and opinions of individual investigators. ${ }^{[7]}$ Most speech pathologists use tests such as WAB, Boston diagnostic aphasia examination, and Mississippi Aphasia Screening test. These batteries are lengthy and require a neurologist or trained speech pathologist to administer. In this study, it took an average of $30 \mathrm{~min}$ to administer WAB depending on the clinical deficits of the patient. Clinical impression was done in fewer than $10 \mathrm{~min}$ for most patients. Therefore, WAB may not be appropriate in setting of an acute stroke, where a rapid evaluation and therapeutic interventions are required. Moreover, it does not necessarily offer a more valid way to classify the aphasic deficits, and there are discrepancies between them. It is a measure of language impairment rather than communication activities of daily living and may not provide a clear description of the underlying language disorder.

One of the limitations of this study was that all patients were examined during the acute phase of stroke and

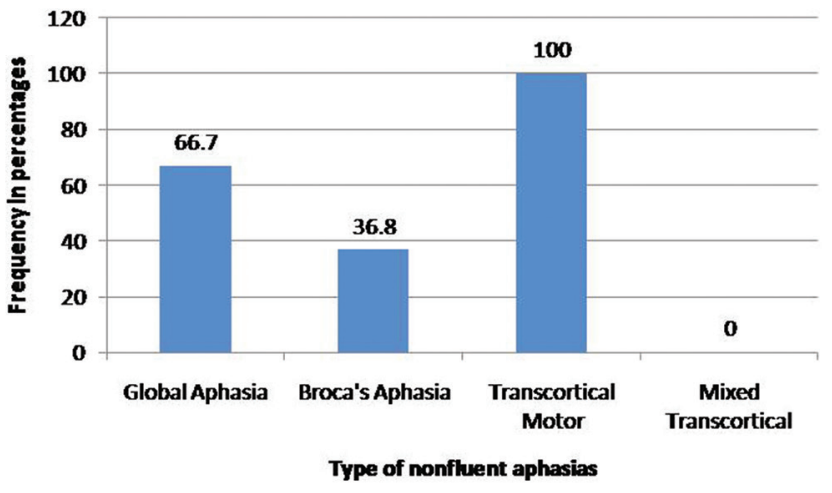

Figure 4: Agreement of clinical impression with Western Aphasia Battery in nonfluent aphasias

some patients of GA, evolved to BA during the process of recovery on follow-up evaluation. Although test for attention span was normal before administering WAB, mild attention deficits in the acute phase of stroke may have had an effect on the AQ and scoring in WAB.

\section{Conclusion}

Clinical impression was fairly reliable, as compared to WAB in assessing the type of aphasia with an overall agreement of $63.4 \%$. However, these data suggest that there may be a discrepancy between WAB classification and clinical impression, especially for the nonfluent and AAs. Therefore, along with WAB, a bedside clinical impression should be done for all the patients to strengthen the description of aphasic deficit. Clinical impression is more appropriate in an acute setting where a rapid diagnosis is required for further management. However, WAB was required to quantify the severity of deficit, which may help in future treatment strategies, accessing prognosis, monitoring progression, and rehabilitation planning.

\section{Acknowledgments}

We would like to thank Dr, Kiran Buddaraju, Dr. Abhinandan K.S., Dr. Abhishek M. and Dr. Lovelina S. for their support in collecting cases and help in preparation of the manuscript. I also thank Ms. Krithika and Ms. Lipsa (speech and language pathologists) for assessing the cases and all the support and help in preparation of this manuscript.

\section{Financial support and sponsorship \\ Nil.}

\section{Conflicts of interest}

There are no conflicts of interest. 


\section{References}

1. Choi-Kwon S, Kim HS, Kwon SU, Kim JS. Factors affecting the burden on caregivers of stroke survivors in South Korea. Arch Phys Med Rehabil 2005;86:1043-8

2. Pedersen PM, Vinter K, Olsen TS. Aphasia after stroke: Type, severity and prognosis. The Copenhagen aphasia study. Cerebrovasc Dis 2004;17:35-43.

3. Wade DT, Hewer RL, David RM, Enderby PM. Aphasia after stroke: Natural history and associated deficits. J Neurol Neurosurg Psychiatry 1986;49:11-6.

4. Shewan CM, Kertesz A. Reliability and validity characteristics of the Western Aphasia Battery (WAB). J Speech Hear Disord 1980;45:308-24.

5. Kertesz A. Aphasia and Associated Disorders: Taxonomy, Localization, and Recovery. New York: Grune \& Stratton; 1979.

6. Chengappa SK, Kumar R. Normative and clinical data on the Kannada version of western aphasia battery (WAB-K). Language in India 2008;8:6

7. Carol SS, Holland AL, Fromm D. Classification of aphasia: WAB type versus clinical impression. In: Clinical Aphasiology Conference. Minneapolis, Minn: BRK Publishers; 1984. p. 48-54 